\title{
POSSIBLE PERIODIC COMPONENTS IN THE FLICKERING OF CH CYG AND MWC 560
}

\author{
M. MIKOLAJEWSKI ${ }^{1}$, T. TOMOV ${ }^{2}$, A. DAPERGOLAS ${ }^{3}$, \\ Y. BELLAS-VELIDIS ${ }^{3}$ \\ 1. Nicolaus Copernicus University, Torun, Poland \\ 2. National Astronomical Observatory Rozhen, Bulgaria \\ 3. National Observatory of Athens, Greece
}

\section{Discussion}

$\mathrm{CH}$ Cyg and MWC 560 are very peculiar symbiotic binaries consisting of an $M$ giant and a white dwarf companion. The systems have many features in common. In particular, both show occasional eruptions with sub-Eddington luminosity, followed by the appearance of high-velocity jets. Recently, Mikołajewski, Mikołajewska \& Tomov (1995) have proposed a model of activity for these systems with a rapidly rotating magnetic white dwarf accreting from the $M$ giant's wind. Two states of activity are possible: propeller and accretor. Although, both stars spend most time in the propeller state, occasional propeller-accretor (and vice versa) transitions may give rise to bipolar outflows and rapid jet formations. Mikołajewski et al. (1995) also proposed to distinguish a new subclass of interacting binaries propeller stars - with CH Cyg and MWC 560 being the prototypes.

One of the most important common features of both stars is the presence of rapid variability (flickering) during active phases with time-scales from minutes to hours, and amplitudes up to $50 \%$ of the blue continuum flux (Mikołajewski et al. 1990; Tomov et al. 1996). However, some periodicities with an amplitude not exceeding $5 \%$ appear relatively frequently.

Our observations were carried out using the $1.2 \mathrm{~m}$ Kryonerian telescope of the Athens National Astronomical Observatory. The $U$ observations of CH Cyg were performed during five nights between 1989 August 1 and 6 . MWC 560 was observed in $U$ and $V$ filters during four nights between 1993 January 18 and 23. The total duration of observations was 14.9 and $8.7 \mathrm{~h}$ for MWC 560 and $\mathrm{CH}$ Cyg, respectively.

Power spectra of both stars' observations show several peaks corresponding to periods of several minutes for $\mathrm{CH}$ Cyg and of tens of min341

A. Evans and J. H. Wood (eds.), Cataclysmic Variables and Related Objects, 341-342. (C) 1996 Kluwer Academic Publishers. Printed in the Netherlands. 
utes for MWC 560. Most of them represent time-scales of variability rather than real periods. They correspond to nearly regularly spaced flares that appear on several occasions and become incoherent after 2-4 cycles. The only peak present on all periodograms appears around $1.9 \mathrm{mHz}$ for $\mathrm{CH} \mathrm{Cyg}$, and around $0.24 \mathrm{mHz}$ for MWC 560, respectively. To determine precisely the periods corresponding to them, we have analysed power spectra of all merged data for both stars. We have found possible coherent oscillations with $P=529.24$ s for $\mathrm{CH}$ Cyg and $P=4225.3$ s for MWC 560 , respectively.

The period found for CH Cyg - of about $530 \mathrm{~s}$ - is close to previously reported values between 498 and $512 \mathrm{~s}$ (Mikołajewski et al. 1990); but it is about five times less than the period recently reported by Hoard (1993). We believe that the $2600 \mathrm{~s}$ period found by Hoard (1993) represents the typical time-scale of a low frequency flickering component rather than a real coherent periodicity. The $70 \mathrm{~m}$ period of MWC 560 belongs to the sharp maximum of possible quasi-periods found in almost fifty different runs of flickering by Tomov et al. (1996). Michalitsianos, Perez \& Shore (1993) have also found a $\sim 1$ h periodicity in their data, very close to our coherent oscillation.

The flickering phenomenon observed in binary systems with orbital periods of several years (Mikołajewski et al. 1995 and refs. therein) can be understood only in terms of instabilities of accretion onto a rapid magnetic rotator. The time-scales and amplitudes of these rapid fluctuations should reflect the interaction between magnetosphere and accreted matter. Since Keplerian rotation near the magnetosphere is close to the period of the rotator one can expect quasi-variability on time-scales close to that period.

Both MWC 560 and $\mathrm{CH}$ Cyg probably contain white dwarfs with magnetic fields of about $10^{7} \ldots 10^{8} \mathrm{G}$. During our observations the stars were at relatively low phases of activity - in the propeller state, when the accretion is stopped by the centrifugal barrier. Simultaneously, a relatively small amount of matter around the rotator makes visible the regions close to the magnetosphere. That is why we can interprete the detected coherent periods as the rotational periods of an accreting white dwarf - or regions very close to it - in both systems.

Acknowledgement. This study was supported by KBN Research Grant No. 2 P304 00706.

\section{References}

Hoard, D.W., 1993, PASP, 105, 1232

Michalitsianos, A.G., Perez, M., Shore, N., 1993, Ap. J., 259, 244

Mikolajewski, M., Mikołajewska, J., Tomov, T., et al., 1990, Acta Astron., 40, 129

Mikolajewski, M., Mikolajewska, J., Tomov, T., 1995, in "Compact Stars in Binaries", Proc. IAU Symp. 165, J. van Paradijs et al. (eds.), in press

Tomov, T., Kolev, D., Ivanov, M., et al., 1996, A\&A, in press 